\title{
Ângulo de Fase e Estado Nutricional de Crianças em Tratamento Antineoplásico
}

doi: https://doi.org/10.32635/2176-9745.RBC.2021v67n2.1234

\author{
Phase Angle and Nutritional Status of Children in Antineoplastic Treatment \\ Ángulo de Fase y Estado Nutricional de los Niños Sometidos a Tratamiento Antineoplásico
}

Isabela Garcia da Cunha Guimarães ${ }^{\text {; }}$ Taísa Alves Silva²; Maísa Tirintan Jordani³; Luciene Alves; Sylvana de Araújo Barros Luz; Camila Bitu Moreno Braga ${ }^{6}$

RESUMO

Introduçáo: $\mathrm{O}$ câncer infantil gera um grande impacto para a saúde pública. A avaliação da composiçáo corporal pela impedância bioelétrica fornece os valores de ângulo de fase, diretamente relacionados a alteraçóes funcionais na membrana celular e que têm sido usados para avaliar o estado nutricional e o prognóstico. Objetivo: Avaliar a correlação entre o ângulo de fase e os parâmetros do estado nutricional de pacientes oncológicos pediátricos em tratamento. Método: Estudo transversal realizado em um hospital público universitário. O estado nutricional foi verificado por meio das medidas de peso, altura, circunferência do braço, dobra cutânea tricipital, índice de massa corporal e circunferência muscular do braço. O ângulo de fase foi determinado pela impedância bioelétrica. Resultados: Foram incluídos 13 pacientes com média de idade de 103,2 $\pm 39,7$ meses, sendo 61,5\% do sexo masculino, com prevalência da leucemia entre os diagnósticos. Constatou-se que a maioria $(53,8 \%)$ dos pacientes estava em eutrofia. Houve forte correlaçáo do ângulo de fase com os parâmetros de massa magra $(r=0,923 ; p=0,000)$, massa corporal celular $(r=0,911 ; p=0,000)$, massa extracelular $(r=0,897 ; p=0,000)$ e peso corporal $(r=0,920 ; p=0,000)$. Conclusáo: Observaram-se correlaçóes expressivas entre o ângulo de fase e os indicadores antropométricos, reforçando a hipótese de que o ângulo de fase pode antecipar a identificação de alteraçôes na composição corporal, possibilitando uma intervenção nutricional precoce e melhor prognóstico.

Palavras-chave: Neoplasias/tratamento farmacológico; Composição Corporal; Impedância Elétrica/uso terapêutico; Estado Nutricional; Criança.

\section{ABSTRACT}

Introduction: Child cancer has a major impact on public health. The evaluation of body composition by bioelectric impedance provides the values of phase angle, causally related to functional changes in the cell membrane which have been used to evaluate nutritional status and prognosis. Objective: To evaluate the correlation between phase angle and parameters of the nutritional status of pediatric oncologic patients under treatment. Method: Cross-sectional study performed in a public university hospital. The nutritional status was evaluated by weight, height, arm circumference, triceps skin fold, body mass index and arm muscle circumference. The phase angle was determined by bioelectric impedance. Results: 13 patients were included with a mean age of $103.2 \pm 39.7$ months, $61.5 \%$ males with prevalence of leukemia in the diagnoses. It was found that most $(53.8 \%)$ of the patients were eutrophic. There was a strong correlation of the phase angle with the parameters of lean mass $(r=0.923 ; p=0.000)$, cellular body mass $(r=0.911 ; p=0.000)$, extracellular mass $(r=0.897 ; p=0.000)$ and body weight $(r=0.920 ; p=0.000)$. Conclusion: Expressive associations between phase angle and other anthropometric indicators were observed, reinforcing the hypothesis that it can anticipate the identification of changes in body composition, allowing early nutritional intervention and better prognosis. Key words: Neoplasms/drug therapy; Body Composition; Electric Impedance/therapeutic use; Nutritional Status; Child.
RESUMEN

Introducción: El cáncer infantil tiene un gran impacto en la salud pública. La evaluación de la composición corporal por impedancia bioeléctrica proporciona los valores del ángulo de fase, directamente relacionados con los cambios funcionales de la membrana celular y que se han utilizado para evaluar el estado nutricional y el pronóstico. Objetivo: Evaluar la correlación entre el ángulo de fase y los parámetros del estado nutricional de los pacientes oncológicos pediátricos en tratamiento. Método: Estudio transversal realizado en un hospital universitario público. El estado nutricional se evaluó por peso, altura, circunferencia del brazo, pliegue de la piel del tríceps, índice de masa corporal y circunferencia muscular del brazo. El ángulo de fase fue determinado por la impedancia bioeléctrica. Resultados: Se incluyeron 13 pacientes con una edad media de 103,2 $\pm 39,7$ meses, de los cuales el $61,5 \%$ eran hombres con prevalencia de leucemia entre los diagnósticos. Se descubrió que la mayoría $(53,8 \%)$ de los pacientes eran eutróficos. Hubo una fuerte correlación del ángulo de fase con los parámetros de masa magra $(r=0,923 ; p=0,000)$, masa corporal celular $(r=0,911 ; p=0,000)$, masa extracelular $(r=0,897 ; p=0,000)$ y peso corporal $(r=0,920 ; p=0,000)$. Conclusión: Se observaron asociaciones expresivas entre el ángulo de fase y otros indicadores antropométricos, lo que refuerza la hipótesis de que puede anticiparse a la identificación de cambios en la composición corporal, lo que permite una intervención nutricional temprana y un mejor pronóstico. Palabras clave: Neoplasias/tratamiento farmacológico; Composición Corporal; Impedancia Eléctrica/uso terapéutico; Estado Nutricional; Ninô.

\footnotetext{
1,2,3 Universidade Federal do Triângulo Mineiro. Uberaba (MG), Brasil. Programa de Residência Integrada Multiprofissional em Saúde. E-mails: isaguimaraes2010@hotmail.com; taisaalves_@hotmail.com; maisajordani@hotmail.com. Orcid iD: https://orcid.org/0000-0003-0988-8141; Orcid iD: https://orcid.org/0000-0001-7725-8470; Orcid iD: https://orcid.org/0000-0002-7952-0062

4,5,6 Universidade Federal do Triângulo Mineiro. Uberaba (MG), Brasil. Instituto de Ciências da Saúde. Departamento de Nutrição. E-mails: luciene.alves@uftm.edu.br sylvana_luz@hotmail.com; camila.braga@uftm.edu.br. Orcid iD: https://orcid.org/0000-0003-4567-3359; Orcid iD: https://orcid.org/0000-0002-2543-0432; Orcid iD: https://orcid.org/0000-0002-5377-1496

Endereço para correspondência: Isabela Garcia da Cunha Guimarães. Rua Professor Enjolrras Vampre, 201, Bloco 1, Apto. 33 - Vila Santa Cândida. São José do Rio Preto (SP), Brasil. CEP 15091-290. E-mail: isaguimaraes2010@hotmail.com
} 


\section{INTRODUÇÃO}

O câncer infantojuvenil (diagnosticado entre as idades de 0 a 19 anos) inclui várias neoplasias malignas e a incidência varia em todo o mundo de acordo com a idade, sexo, etnia e geografia. Apesar de ser um tipo de câncer considerado raro, quando comparado com o adulto, o número de casos novos nessa faixa etária representa um grande impacto para a saúde pública, pois as taxas de mortalidade costumam ser altas, além dos importantes efeitos colaterais gerados pelo tratamento ${ }^{1}$.

O câncer em crianças e adolescentes, quando confrontado com as demais neoplasias, corresponde de $1 \%$ a $4 \%$ dos casos ${ }^{2}$. Segundo a American Cancer Society ${ }^{3}$, as leucemias são os tipos mais prevalentes (28\%), seguidas dos de sistema nervoso central (26\%) e dos linfomas (8\%). No Brasil, estima-se que, entre o período de 2020-2022, manifestem-se 4.310 novos casos na população masculina e 4.150 na feminina, por ano ${ }^{4}$.

A perda progressiva de peso e a consequente depleção nutricional sáo comumente encontradas em pacientes oncológicos e têm impacto direto para um desfecho desfavorável. Nesse sentido, detectar precocemente as variaçôes nutricionais é de extrema importância para prevenção de alteraçóes morfológicas e funcionais, garantindo desenvolvimento e crescimento adequado em crianças e adolescentes ${ }^{5}$. A identificação rápida e simples do risco nutricional por meio dos instrumentos de triagem e a avaliação da condição nutricional devem ser açôes primárias no tratamento desses pacientes ${ }^{6}$.

A antropometria avalia a composição corporal por intermédio da aferição de parâmetros de peso, estatura, dobras cutâneas e circunferências. $\mathrm{Na}$ avaliação nutricional de pacientes oncológicos pediátricos, deve-se atentar para a presença de edema, ascite e massa tumoral, que podem levar a uma equivocada classificação do estado nutricional ${ }^{7}$. A impedância bioelétrica (BIA) é um método de avaliação da composição corporal considerado simples, de fácil aplicação, baixo custo, não invasivo e rápido, além de poder ser realizado à beira do leito.

A partir da BIA. é possível determinar componentes corporais, como massa magra, massa gorda e água, além de fornecer também o ângulo de fase $(\mathrm{AF})^{8}$. O AF é uma medida obtida por meio da relaçáo entre a resistência (R); isto é, a oposição dos tecidos à corrente elétrica, e a reactância $(\mathrm{Xc})$, que representa a capacitância das membranas celulares ${ }^{8}$. O valor de AF está relacionado a alteraçóes funcionais na membrana da célula, com reflexo nos tecidos corporais e distribuição hídrica entre os meios intra e extracelulares, sendo cada vez mais encorajado para avaliar o estado nutricional e o prognóstico, inclusive em pacientes oncológicos. Entretanto, até o momento, são escassos na literatura científica os estudos que avaliam o papel do AF em crianças com câncer ${ }^{9,10}$.

Neste contexto, o objetivo do presente estudo foi avaliar a correlaçáo entre AF e parâmetros do estado nutricional de pacientes pediátricos em tratamento antineoplásico.

\section{MÉTODO}

Trata-se de um estudo transversal que incluiu 13 pacientes pediátricos diagnosticados com neoplasias e em tratamento antineoplásico. Todos os pacientes recrutados por amostra de conveniência passaram por uma intervenção pontual. Os participantes avaliados estavam acompanhados pelo responsável legal e ambos os dois foram informados sobre os objetivos e procedimentos do estudo e assinaram o Termo de Assentimento Livre e Esclarecido (Tale). Os dados foram coletados durante o período de julho a setembro de 2018. As informaçóes referentes a data de nascimento, gênero, tipo de tumor, estadiamento tumoral, ciclo de quimioterapia, quimioterápico e tempo de diagnóstico foram coletadas dos prontuários eletrônicos dos pacientes.

O projeto foi aprovado pelo Comitê de Ética em Pesquisa institucional sob o número CAAE: 81081717.0 .0000 .5154 e seguiu a Resolução n. ${ }^{\circ}$ $466 / 2012^{11}$. Os critérios de inclusão foram indivíduos entre 0 e 19 anos, de ambos os sexos, com diagnóstico de neoplasias em tratamento quimioterápico e/ou radioterápico. Foram excluídos pacientes com prontuários incompletos, casos de recidiva e com outras patologias de base.

Para avaliação da composição corporal, foi realizada a BIA (Byodinamics ${ }^{\oplus}$, modelo 450, marca TBW Brasil), respeitando as recomendaçóes do fabricante. Para a avaliação antropométrica, foram aferidos: peso $(\mathrm{kg})$, altura $(\mathrm{cm})$, circunferência do braço $(\mathrm{CB})(\mathrm{cm})$, e dobra cutânea tricipital (DCT) $(\mathrm{mm})$, com cálculo do índice de massa corporal (IMC) $\left(\mathrm{kg} / \mathrm{m}^{2}\right)$ e da circunferência muscular do braço $(\mathrm{CMB})(\mathrm{cm})$, seguindo as orientaçóes do Manual de Avaliação Nutricional da Sociedade Brasileira de Pediatria ${ }^{12}$. Os voluntários foram avaliados conforme os indicadores antropométricos de peso para idade (P/I) (0 a 10 anos), peso para estatura (P/E) (0 a 5 anos), estatura para idade (E/I) (0 a 19 anos), índice de massa corporal para idade (IMC/I) (0 a 19 anos) e, classificados em escore $Z$ pelas curvas de crescimento da Organização Mundial da Saúde (OMS) de 2006/2007 (softwares WHO Anthro versão 3.2.2 e WHO Anthro Plus 1.0.4). Para os dados de CB, CMB e DCT, foram usados, como referências para classificação, os padrôes de Frisancho ${ }^{13}$. 
Para análise estatística, foi empregado o programa Statistics $6.0^{\circ}$ (StatSoft Incorporation, Tulsa, Ok, USA). Para todas as análises, foi fixado um nível de significância de 5\%. O teste de Shapiro-Wilks foi aplicado para testar a normalidade da distribuiçáo dos dados obtidos. As variáveis foram apresentadas como média e desvio-padrão para variáveis paramétricas e, como mediana, valores mínimos e máximos para as não paramétricas. A comparação entre grupos (sexos) foi feita pelo teste $t$ de Student. A correlação de Pearson foi utilizada para avaliar presença de correlação entre os diversos parâmetros do estudo, sendo considerada uma correlação fraca valores de $r$ entre 0,1 e 0,3 , moderada entre 0,4 e 0,6 e forte entre 0,7 e $1^{14}$.

\section{RESULTADOS}

A casuística constou de 13 pacientes, sendo oito do sexo masculino $(61,5 \%)$ e cinco do sexo feminino (38,5\%), com média de idade de 103,2 $\pm 39,7$ meses. Em relação ao diagnóstico clínico, oito pacientes apresentavam leucemia linfocítica aguda, um glioma de baixo grau irressecável, um linfoma não Hodgkin, um linfoma Hodgkin, um osteossarcoma e uma histiocitose de células Langerhans. Em relação ao tipo de tratamento, todos os pacientes estavam em ciclo de quimioterapia e dois pacientes em radioterapia. Os pacientes tinham uma mediana de tempo de diagnóstico até a intervenção de nove meses, sendo um mês o mínimo e 21 o máximo.
Três pacientes tinham doenças associadas, tais como hipertensão, síndrome depressiva e suspeita diagnóstica de síndrome de Cushing.

Os dados relativos à antropometria e os parâmetros obtidos pela BIA estáo apresentados na Tabela $1 . \mathrm{Na}$ classificação do IMC/I, sete $(53,8 \%)$ pacientes estavam eutróficos, um $(7,7 \%)$ com risco de sobrepeso, três $(23,1 \%)$ com sobrepeso e dois $(15,4 \%)$ com obesidade. Todos os pacientes apresentaram estatura adequada para a idade.

Os pacientes apresentaram uma média de porcentagem de adequação de $114,8 \%$ para CB e $117 \%$ para DCT, considerando os valores de percentil 50, para sexo e idade $^{13}$. A correlaçáo dos valores de AF com os demais parâmetros do estado nutricional está demonstrada na Tabela 2. Apenas as variáveis $\mathrm{CMB}, \mathrm{CB}$ e DCT não apresentaram correlação forte com o AF.

\section{DISCUSSÃO}

No presente estudo, observou-se que nenhum paciente estava com desnutrição pelo critério de IMC/I. Além disso, o AF apresentou forte correlaçáo com outros parâmetros antropométricos: massa magra, massa celular corporal (MCC), massa extracelular (ME), água total e peso corporal. Estudos vêm demostrando a ação do AF como indicador do estado nutricional nessa população, baixos valores de $\mathrm{AF}$ indicariam risco nutricional mesmo com IMC compatível com eutrofia. Alteraçôes em nível celular

Tabela 1. Distribuição das variáveis de composição corporal de todos os voluntários do estudo e de acordo com o sexo

\begin{tabular}{lccc} 
& $\begin{array}{c}\text { Todos } \\
(\mathbf{n = 1 3 )}\end{array}$ & $\begin{array}{c}\text { Sexo feminino } \\
(\mathbf{n = 5})\end{array}$ & $\begin{array}{c}\text { Sexo masculino } \\
(\mathbf{n}=\mathbf{8})\end{array}$ \\
\hline Peso corporal $(\mathrm{kg})$ & $30,2 \pm 11,9$ & $26,1 \pm 8,4$ & $32,7 \pm 13,1$ \\
CB $(\mathrm{cm})$ & $20,1 \pm 3,8$ & $19,8 \pm 2,2$ & $20,3 \pm 4,6$ \\
DCT $(\mathrm{mm})$ & $14,4 \pm 4,8$ & $13,2 \pm 1,78$ & $15,1 \pm 6,0$ \\
CMB $(\mathrm{cm})$ & $15,6 \pm 2,9$ & $15,6 \pm 2,0$ & $15,5 \pm 3,4$ \\
IMC $\left(\mathrm{kg} / \mathrm{m}^{2}\right)$ & $17,7 \pm 3,0$ & $17,5 \pm 1,95$ & $18,6 \pm 3,2$ \\
Massa magra (kg) & $23,6 \pm 7,9$ & $21,4 \pm 8,3$ & $25,1 \pm 7,8$ \\
Massa magra (\%) & $80,2 \pm 7,8$ & $80,9 \pm 5,3$ & $79,7 \pm 9,4$ \\
Massa gorda (kg) ${ }^{1}$ & $7,1 \pm 4,9$ & $4,9 \pm 1,7$ & $8,5 \pm 5,7$ \\
Massa gorda (\%) & $21,6 \pm 7,7$ & $18,8 \pm 5,1$ & $23,3 \pm 8,8$ \\
Água (L) & $17,6 \pm 5,2$ & $16,4 \pm 6,1$ & $18,9 \pm 4,8$ \\
Água total (\%) & $75,8 \pm 4,4$ & $77,5 \pm 2,1$ & $74,7 \pm 5,3$ \\
MCC & $10,9 \pm 3,4$ & $10,3 \pm 4,1$ & $10,9 \pm 4,2$ \\
ME & $12,8 \pm 4,6$ & $11,3 \pm 3,1$ & $14 \pm 4,7$ \\
Ângulo de fase $\left(^{\circ}\right)$ & $4,6 \pm 1,0$ & $4,7 \pm 1,4$ & $4,5 \pm 0,7$ \\
\hline
\end{tabular}

Legendas: CB: circunferência do braço; DCT: dobra cutânea tricipital; CMB: circunferência muscular do braço, IMC: índice de massa corporal; MCC: massa celular corporal; ME: massa extracelular; ${ }^{1}$ : $\mathrm{p}<0,05$ pelo test t Student. 
Tabela 2. Correlação de Pearson do ângulo de fase vs. parâmetros do estado nutricional de todos os voluntários do estudo $(n=13)$

\begin{tabular}{lcc} 
& Valor de $\mathbf{r}$ & Valor de $\mathbf{p}$ \\
\hline Massa magra $(\mathrm{kg})$ & 0,923 & $0,000^{*}$ \\
Massa gorda $(\mathrm{Kg})$ & 0,734 & $0,004^{*}$ \\
MCC & 0,911 & $0,000^{*}$ \\
ME & 0,897 & $0,000^{*}$ \\
ME/MCC & 0,919 & $0,024^{*}$ \\
Água total (L) & 0,920 & $0,000^{*}$ \\
Água total (\%) & $-0,773$ & $0,002^{*}$ \\
Peso atual (kg) & 0,920 & $0,000^{*}$ \\
CMB (cm) & 0,569 & $0,042^{*}$ \\
CB (cm) & 0,618 & $0,024^{*}$ \\
DCT (mm) & 0,471 & 0,104 \\
\hline
\end{tabular}

Legendas: MCC: massa celular corporal; ME: massa extracelular; CMB: circunferência muscular do braço; DCT: dobra cutânea tricipital; CB: circunferência do braço; ${ }^{*}$ Valor de p considerado significativo $<0,05$.

ocorrem à frente das alterações teciduais, detectáveis pelos métodos antropométricos tradicionais, evidenciando a relevância do AF na prática clínica como instrumento de triagem e indicador de prognóstico ${ }^{15,16}$.

A BIA e consequentemente os valores de AF têm sido objetivo de estudos nos últimos anos como instrumento de valor prognóstico, nutricional, de função de membrana celular ou indicador de saúde em várias condições clínicas e em adultos e crianças ${ }^{15,17,18}$. Sua associação com as demais ferramentas de avaliação, como a antropometria, em pacientes sob risco nutricional, pode levar a uma intervenção mais precisa?.

$\mathrm{O}$ valor médio de AF encontrado nos voluntários deste estudo foi de $4,5^{\circ}$. Até o presente momento, não existem valores de referência estabelecidos na literatura de AF para população de crianças brasileiras saudáveis para comparação. Um trabalho realizado por De Palo et al. ${ }^{17}$, com 2.044 crianças saudáveis entre 10 e 15 anos, encontrou uma variação do AF maior do que o encontrado no presente estudo (entre $5,7^{\circ}$ e $6,2^{\circ}$ ).

Em outros estudos, valores de $\mathrm{AF}$ entre $0,7^{\circ}$ e $3,1^{\circ}$ foram encontrados em uma população de pacientes pediátricos com desnutriçãoo ${ }^{18,19}$. A possível associação entre o $\mathrm{AF}$ e os indicadores antropométricos pode ser explicada pelo fato de o AF refletir a função da membrana celular e da MCC, predominantemente constituída por músculos. Quando encontrados valores baixos de AF, pode-se sugerir morte celular ou alteração na integridade da membrana celular, o contrário indica membranas celulares intactas 9 . Desse modo, sugere-se que menor valor de AF pode ser atribuído ao processo de sarcopenia já relatado em pacientes oncológicos e desnutridos ${ }^{20}$.
Ainda são poucos os estudos na literatura que abordam o papel do AF na população infantil em tratamento antineoplásico. Garofalo et al. ${ }^{21}$ demonstraram em estudo com 33 pacientes oncológicos pediátricos, com média de idade de 10 anos, a presença de baixos valores de $\mathrm{AF}$ quando comparados com os encontrados em crianças saudáveis $\left(5,5^{\circ} v\right.$ s. $4,8^{\circ}$, respectivamente). Nos pacientes críticos, esses baixos valores de AF foram associados a um precário estado nutricional, piores desfechos clínicos e alta mortalidade ${ }^{21}$.

A associação entre o AF e o tempo de internação e mortalidade de pacientes pediátricos críticos foi avaliada em estudo com 247 voluntários internados em unidades de terapia intensiva. Uma maior sobrevida foi encontrada em crianças com AF maior do que $2,8^{\circ}$. Notou-se também que aquelas com menores valores de AF tinham maior tempo de hospitalização ${ }^{22}$.

Em estudo conduzido por Pereira et al. ${ }^{23}$, em um hospital universitário brasileiro com crianças em tratamento quimioterápico, constituinte de dois grupos: grupo 1 (leucemias, média de 92 meses de idade) e grupo II (linfomas e tumores sólidos, média de 62 meses de idade), foi realizada BIA em três momentos: antes da primeira sessão de quimioterapia, na metade do protocolo de tratamento, ao final da fase de indução em leucemias, e uma semana após a aplicação da última quimioterapia para tumores sólidos. Semelhante aos nossos achados, o grupo I obteve valores de $\mathrm{AF}$ de $4,6^{\circ} \pm 1,2,4,5^{\circ} \pm 1,1$ e $4,5^{\circ} \pm$ 0,7 , nos três tempos, respectivamente. Para os tumores sólidos, as médias de AF foram 5, $1^{\circ} \pm 1,4,4,8^{\circ} \pm 0,9$ e $4,6^{\circ} \pm 0,7$. Não houve identificação de alteração do $A F$ no decorrer do tratamento em nenhum dos grupos, porém, ao observar individualmente os pacientes dos diferentes grupos, notou-se que a maioria apresentou reduçáo dos valores de $\mathrm{AF}$ ao longo do tratamento, independentemente do tipo de tumor ${ }^{23}$.

A partir dessa redução dos valores de AF, encontrada em diferentes estudos, pode-se levantar a hipótese de que os pacientes podem estar em risco de desnutrição mesmo quando seus valores de IMC estão compatíveis com eutrofia, o que se justifica pelo fato de as alteraçóes em nível celular ocorrerem à frente das de nível tecidual (que pode ser detectado pelos métodos habituais para o diagnóstico nutricional, como a antropometria) ${ }^{24}$. Entretanto, os quimioterápicos também podem promover baixos valores de $\mathrm{AF}$, uma vez que a membrana celular atua na eliminação das drogas administradas e o AF depende diretamente de sua integridade ${ }^{25}$.

O estudo possui algumas limitaçóes, como o fato de ser transversal, impossibilitando inferir relações causais e não possuir grupo controle. Outro ponto é o tamanho da casuística, uma vez que se trata de uma amostragem 
feita por conveniência, já que o serviço não é referência em tratamento oncológico pediátrico.

\section{CONCLUSÃO}

Pacientes pediátricos em tratamento antineoplásico apresentaram fortes correlaçôes entre AF e parâmetros antropométricos de massa magra, MCC/ME e peso corporal. A BIA é um método de avaliação não invasivo e de fácil aplicação e a detecção do AF pode identificar precocemente os pacientes em risco nutricional, permitindo uma intervenção imediata.

\section{CONTRIBUIÇÕES}

Todas as autoras contribuíram na concepção e/ ou no planejamento do estudo; na obtençáo, análise e interpretação dos dados; assim como na redação e revisão crítica; e aprovaram a versão final a ser publicada.

\section{DECLARAÇÃO DE CONFLITO DE INTERESSES}

Nada a declarar.

\section{FONTES DE FINANCIAMENTO}

Não há.

\section{REFERÊNCIAS}

1. Instituto Nacional de Câncer José Alencar Gomes da Silva. Consenso nacional de nutrição oncológica. 2. ed. rev. ampl. atual. Rio de Janeiro: INCA; 2016.

2. American Cancer Society. Cancer facts \& figures [Internet]. Atlanta: American Cancer Society; 2014 [cited 2020 June 21]. Available from: https://www.cancer.org/ research/cancer-facts-statistics/all-cancer-facts-figures/ cancer-facts-figures-2014.html

3. American Cancer Society. Cancer facts \& figures [Internet]. Atlanta: American Cancer Society; 2019 [cited 2020 June 21]. Available from: https://www.cancer. $\mathrm{org} /$ content/dam/cancer-org/research/cancer-facts-andstatistics/annual-cancer-facts-and-figures/2019/cancerfacts-and-figures-2019.pdf

4. Instituto Nacional de Câncer José Alencar Gomes da Silva. Estimativa 2020: incidência de câncer no Brasil [Internet]. Rio de Janeiro: INCA; 2019 [acesso 2020 jun 21]. Disponível em: https://www.inca.gov.br/sites/ufu. sti.inca.local/files//media/document//estimativa-2020incidencia-de-cancer-no-brasil.pdf

5. Alberti DN, Ascari RA, Schirmer EM. Parâmetros bioquímicos e estado nutricional de pacientes cirúrgicos com câncer gastrointestinal. Rev Col Bras. 2020;47:e20202512. doi: http://doi.org/10.1590/01006991e-20202512

6. Coruja MK, Steemburgo T. Estado nutricional e tempo de internação de pacientes adultos hospitalizados com diferentes tipos de câncer. BRASPEN J. 2017;32(2):114-8.

7. Viani KHC. Estado nutricional e sobrevida global de crianças e adolescentes com câncer acompanhados pelo serviço de nutrição [tese]. São Paulo (SP): Universidade de São Paulo; 2019.

8. Nascimento ACS, Pinho CPS, Santos ADA, et al. Ângulo de fase e indicadores de risco do (e indicadores de riesgo del) estado nutricional em pacientes pré-cirúrgicos. Salud(i) Ciencia. 2018;23:134-40. doi: http://www.doi. org/10.21840/siic/154251

9. Norman K, Stobäus N, Pirlich M, et al. Bioelectrical phase angle and impedance vector analysis - clinical relevance and applicability of impedance parameters. Clin Nutr. 2012;31(6):854-61.doi: https://doi.org/10.1016/j. clnu.2012.05.008

10. Pena NF. Associação do ângulo de fase padronizado com estado nutricional e desfechos clínicos em pacientes cirúrgicos oncológicos [dissertação]. Belo Horizonte (MG): Universidade Federal de Minas Gerais; 2016.

11. Conselho Nacional de Saúde (BR). Resoluçấo no 466, de 12 de dezembro de 2012. Aprova as diretrizes e normas regulamentadoras de pesquisas envolvendo seres humanos. Diário Oficial da União, Brasília, DF; 2013 jun. 13. Seção I, p. 59.

12. Sociedade Brasileira de Pediatria, Departamento de Nutrologia. Manual de orientação: avaliaçáo nutricional da criança e adolescente. Rio de Janeiro: SBP; 2009.

13. Frisancho AR. Anthropometric standards for the assessment of growth and nutritional status. Ann Arbor (MI): University of Michigan Press; 1990.

14. Dancey C, Reidy J. Estatística sem matemática para psicologia: usando SPSS para Windows. 3. ed. Porto Alegre: Artmed; 2006.

15. Pileggi VN, Scalize ARH, Camelo Junior JS. Phase angle and World Health Organization criteria for the assessment of nutritional status in children with osteogenesis imperfecta. Rev Paul Pediatr. 2016;34(4):484-8. doi: https://doi.org/10.1016/j.rppede.2016.03.010

16. Pereira MME, Wiegert EVM, Oliveira LC, et al. Ângulo de fase e estado nutricional em indivíduos com câncer avançado em cuidados paliativos. Rev Bras Canc. 2019;65(1):e-02272. doi: http://doi. org/10.32635/2176-9745.RBC.2019v65n1.272

17. De Palo T, Messina G, Edefonti A, et al. Normal values of the bioelectrical impedance vector in childhood and puberty. Nutrition. 2000;16(6):417-24. doi: http://doi. org/10.1016/s0899-9007(00)00269-0

18. Azevedo ZMA, Silva DR, Dutra MVP, et al. Associação entre ângulo de fase, PRISM I e gravidade da sepse. Rev 
Bras Ter Intensiva. 2007;19(3):297-303. doi: https://doi. org/10.1590/S0103-507X2007000300005

19. Costa GLOB. Ângulo de fase enquanto indicador de estado nutricional no câncer do trato digestório [dissertação]. Salvador (BA): Universidade Federal da Bahia; 2012.

20. Marriott CJC, Beaumont LF, Farncombe TH, et al. Body composition in long-term survivors of acute lymphoblastic leukemia diagnosed in childhood and adolescence: a focus on sarcopenic obesity. Cancer. 2018;124(6):1225-31. doi: https://doi.org/10.1002/ cncr.31191

21. Garofalo A, Guedes KJT, Maia-Lemos P. Low phase angle (PA) values of the electrical bioimpedance analysis (BIA) in pediatrics patients with cancer. Pediatr Blood Cancer. 2017;64S402.

22. Zamberlan P, Feferbaum R, Doria Filho U, et al. Bioelectrical impedance phase angle and morbidity and mortality in critically III children. Nutr Clin Pract. 2019;34(1):163-71. doi: https://doi.org/10.1002/ ncp. 10201

23. Pereira ACV, De Souza A, Dudeque MPA, et al. Avaliação do ângulo de fase antes, durante e após tratamento quimioterápico em crianças e adolescentes. Nutr Clin Diet Hosp. 2018;38(1):156-9. doi: https://doi. org/10.12873/381ACoradine

24. Guida B, De Nicola L, Pecoraro P, et al. Abnormalities of bioimpedance measures in overweight and obese hemodialyzed patients. Int J Obes Relat Metab Disord. 2001;25(2):265-72. doi: https://doi.org/10.1038/ sj.ijo.0801475

25. Ge Y, Haska CL, LaFiura K, et al. Prognostic role of the reduced folate carrier, the major membrane transporter for methotrexate, in childhood acute lymphoblastic leukemia: a report from the Children'S Oncology Group. Clin Cancer Res. 2007;13(2 Pt 1):451-7. doi: https:// doi.org/10.1158/1078-0432.CCR-06-2145 